\title{
BANDGAP PROPERTIES IN LOCALLY RESONANT PHONONIC CRYSTAL DOUBLE PANEL STRUCTURES WITH PERIODICALLY ATTACHED PILLARS
}

\author{
Denghui Qian, Zhiyu Shi \\ State Key Laboratory of Mechanics and Control of Mechanical Structures, College of Aerospace Engineering, \\ Nanjing University of Aeronautics and Astronautics, Nanjing, Jiangsu, China \\ e-mail: qdhsd318@163.com; zyshi@nuaa.edu.cn (correspongding author)
}

\begin{abstract}
The locally resonant (LR) phononic crystal double panel structure made of a two-dimensional periodic array of a two-component cylindrical LR pillar connected between the upper and lower plates is proposed, and the bandgap properties of the structure are investigated theoretically in this paper. The band structures, displacement fields of eigenmodes and transmission power spectrums of the corresponding $8 \times 8$ finite structure are calculated by the finite element method. Numerical results and further analysis demonstrate that a band gap with a low starting frequency and a wide band width is opened by the coupling between dominant vibrations of the pillars and plate modes of the upper and lower plates when the vibration source and the receiver are considered on different sides of the structure. By comparing the band structures and displacement fields of the double panel and those of the single plate with the same parameters, many common characteristics are displayed. Then, the influence of geometrical parameters on the band gap are studied and understood with the help of a simple 'spring-mass' model.
\end{abstract}

Keywords: bandgap property, phononic crystal double panel, band structure, displacement field, transmission power spectrum

\section{Introduction}

Due to the advantageous sound-insulated property, double panels are extensively used as the containment structures in many areas such as marine, transport, aerospace engineering and civil construction projects (Carnael and Fuller, 2004; Pietrzko and Mao, 1992). As it is well-known, vibrations are mostly propagated along containment structures from vibration sources, and structural noises are produced by radiation of the vibrations. The proposition of the phononic crystal concept provides a new idea for the investigation on theory about vibration insulation and noise reduction. Over the past two decades, the propagation of elastic waves in phononic crystals has attracted a lot of attention which mainly focuses on calculation methods and bandgap properties, but the application researches particularly on the field of vibration insulation and noise reduction are still immature. Brag scattering (Saindou et al., 2002; Sigalas and Economou, 1992; Vasseur etal, 2002; Zhang et al., 2003) and locally resonant (Goffaux et al., 2004; Hirsekorn et al., 2004; Ho et al., 2003; Liu et al., 2000) are developed as the two main mechanisms for the creation of band gaps, in which the frequency range of band gaps based on the first mechanism is almost two orders of magnitude higher than that based on the second mechanism (Liu et al., 20007). Hence, the studies on double panel structures with the design idea of locally resonant phononic crystal introduced provide a new idea for restraining structural vibration and reducing noise in the unmanageable low frequency region (Hsu, 2011; Oudich et al., 2011; Qian and Shi, 2017; Xiao et al., 2017) of some industrial products. 
For now, bandgap properties of double panel structures with the design idea of a locally resonant phononic crystal introduced have rarely been studied. However, such an idea has been widely implemented in single plates recently. By etching holes periodically in a solid matrix plate and then filling them with scatters, the so-called filled-in system is formed. By stubbing resonant units periodically onto free surfaces of the plate, a stubbed-on system is formed (Ma et al., 2014). Hsu and Wu (2007) and Xiao et al. (2008) investigated vibration of band gaps of epoxy base plates with filled-in rubber resonant units and filled-in rubber-coated heavy mass resonant units by using the plane wave expansion method, respectively. Similarly, the three-component and two-component stubbed-on systems constructed by periodically depositing rubber stubs with and without $\mathrm{Pb}$ capped on the surface of the base plate were studied by using the finite element method by Oudich et al. (2010). Besides, Xiao et al. (2012) researched flexural wave propagation and vibration transmission in a locally resonant thin plate with a two-dimensional periodic array of attached spring-mass resonators, which can be regarded as a simplified model of the stubbedon system. Zhao et al. (2016) proposed a double-vibrator (rubber-steel-rubber-steel layers) threecomponent pillared PC plate on the basis of the traditional uni-vibrator (rubber-steel layers) three-component pillared PC plate and studied propagation characteristics of band gaps of flexural vibration and longitudinal vibration in the two-layer stubbed-on system. By revisiting the filled-in and stubbed-on structures, Ma et al. (2014) proposed a new structure with threelayered spherical resonant units, which opens a large sub-wavelength full band gap. By combining the filled-in and stubbed-on units, Li et al. (2015) investigated propagation characteristics of Lamb waves in a locally resonant phononic crystal single plate with the combined resonant unit. Based on this, Li et al. (2016) further researched the expansion of locally resonant complete band gaps in two-dimensional phononic crystals using a double-sided stubbed composite PC plate with composite stubs. Recently, Qian and Shi (2016) investigated the propagation characteristics of flexural waves in the locally resonant phononic crystal double panel structure made of a two-dimensional periodic array of a spring-mass resonator surrounded by $n$ springs connected between the upper and lower plates.

In this paper, we investigate propagation characteristics of flexural vibration and longitudinal vibration in a locally resonant double panel structure consisting of a two-layer uniform thin plate with periodically attached cylindrical LR pillars in the cavity. At first, the band structures, displacement fields of eigenmodes and transmission power spectrums of the corresponding finite structure are calculated to study the formation mechanisms of the band gap. Then, a comparison between the band structure of the double panel and that of the single plate with the same parameters is displayed. Further, the influence of the geometrical parameters such as height of the rubber layer in the pillar, height of the $\mathrm{Pb}$ layer, radius of the pillar, thickness of the base plate and the lattice constant on the band gap of the proposed structure are discussed.

(a)

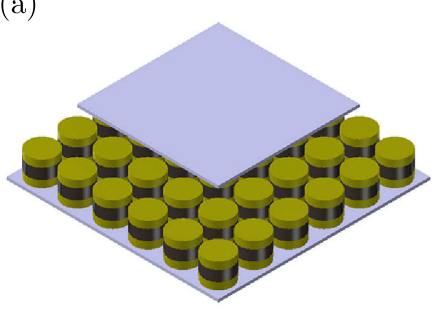

(b)

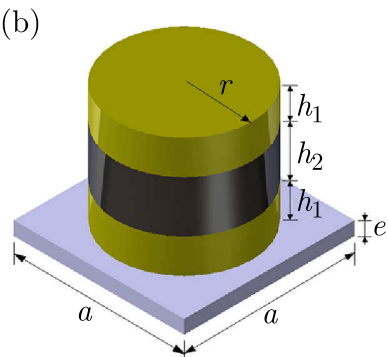

(c)

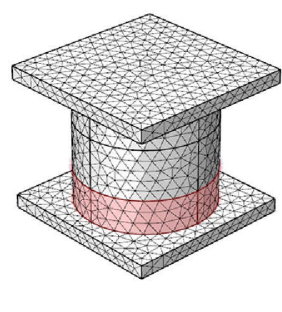

(d)

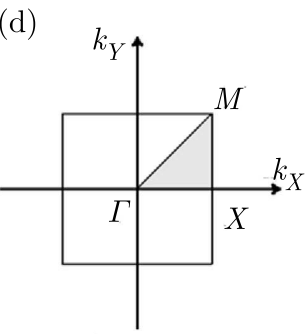

Fig. 1. (a) The LR phononic crystal double panel structure with periodically attached pillars, (b) its unit cell (upper plate is ignored), $a$ is the lattice constant, $e$ is thickness of the base plates, $r$ is radius of the pillar, $h_{1}$ is height of rubber layer in the pillar, and $h_{2}$ is height of $\mathrm{Pb}$ layer in the pillar,

(c) meshing of the unit cell, (d) and the irreducible first Brillouin zone (1BZ) 


\section{Model and method}

As shown in Fig.1a, the studied system is constructed by periodically depositing the two-component cylindrical LR pillars squarely onto the surfaces of the upper and lower thin plates. The material of the first and last layers in the pillar is rubber, and the materials of the plates and the middle layer in the pillar are epoxy and $\mathrm{Pb}$, respectively. The lattice constant, thickness of the base plates, radius of the pillar, and heights of different layers in the pillar in a unit cell are denoted by $a, e, r, h_{1}$ and $h_{2}$, which can be seen in Fig. 1b. Table 1 displays the material parameters used in the calculation, and all materials are assumed to be elastically isotropic.

Table 1. Material parameters used in calculations

\begin{tabular}{|c|c|c|c|}
\hline Material & $\begin{array}{c}\text { Mass density } \\
{\left[\mathrm{kg} / \mathrm{m}^{3}\right]}\end{array}$ & $\begin{array}{c}\text { Young's modulus } \\
{\left[10^{10} \mathrm{~N} / \mathrm{m}^{2}\right]}\end{array}$ & $\begin{array}{c}\text { Poission's } \\
\text { ratio }\end{array}$ \\
\hline \hline Epoxy & 1180 & 0.435 & 0.368 \\
\hline Rubber & 1300 & $1.175 \mathrm{e}-5$ & 0.469 \\
\hline $\mathrm{Pb}$ & 11600 & 4.08 & 0.37 \\
\hline
\end{tabular}

The band structure of the proposed system is calculated by the finite element method (FEM), which is implemented by adopting the commercial software, COMSOL Multiphysics. For the mesh elements, the default tetrahedral mesh provided by the software is used and the meshing of the unit cell is shown in Fig. 1c. From the figure, we can see only that one unit cell is taken into consideration, which can be attributed to the periodicity of the structure. The stress-free boundary conditions are applied to the free surfaces, and the periodic boundary conditions according to the Bloch-Floquet theorem are used for the interfaces between the nearest unit cells (Hsu and Wu, 2007; Xiao et al., 2008)

$$
u_{i}(x+a, y+a)=u_{i}(x, y) \mathrm{e}^{-\mathrm{i}\left(k_{x} a+k_{y} a\right)} \quad i=x, y, z
$$

where $u_{i}$ denotes the elastic displacement along the $x-, y$ - and $z$-direction, respectively, when $i$ equals to $x, y$ and $z ; k_{x}$ and $k_{y}$ are components of the Bloch wave vector limited in the irreducible first Brillouin zone (1BZ), as shown in Fig. 1d.

\section{Numerical results and analyses}

\subsection{Band structures, eigenmodes and transmission power spectrums}

In this Section, the band structure of the proposed double panel structure is calculated, as shown in Fig. 2b. The parameters are as follows: $a=0.1 \mathrm{~m}, e=0.005 \mathrm{~m}, r=0.04 \mathrm{~m}$, $h_{1}=0.01 \mathrm{~m}$, and $h_{2}=0.03 \mathrm{~m}$. To verify the accuracy of the calculated result, the transmission power spectrums of the flexural vibration and the longitudinal vibration in the corresponding finite system are displayed in Figs. 2a and 2c, separately. In this work, the finite double panel structure is made of $8 \times 8$ unit cells and the excitation point is picked on one end of the lower plate as well as the response point is picked on the other end of the upper plate, as shown in Fig. 3.

From Fig. 2b, a narrow complete band gap is observed between $72 \mathrm{~Hz}$ and $83 \mathrm{~Hz}$. But from Figs. 2a and 2c, the frequency range of the attenuation in the transmission power spectrum is very wide no matter the finite structure is vibrated flexurally or longitudinally, which should have matched well with the frequency range of the band gap. To find more attenuation characteristics, both the response point and the excitation point are picked on the lower plate, and the transmission power spectrum of the longitudinal vibration is shown in Fig. 2d. From it, 
(a)

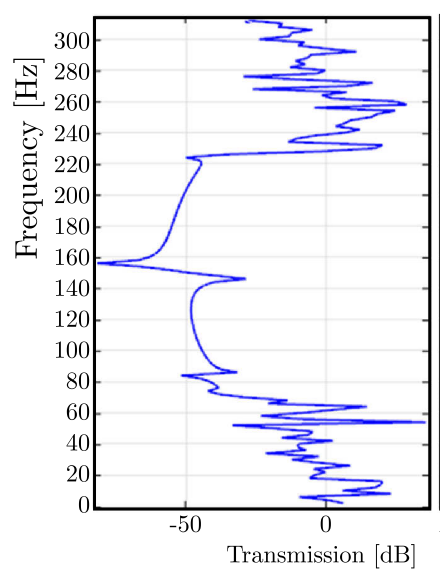

(b)

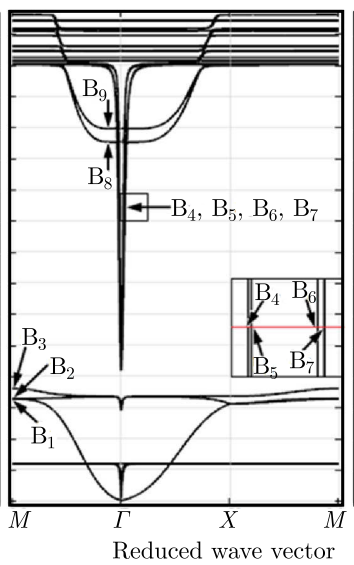

(c)

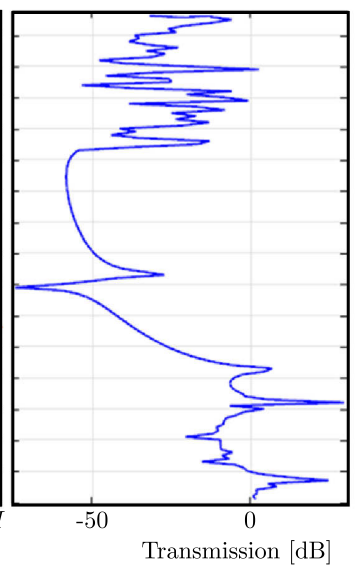

(d)

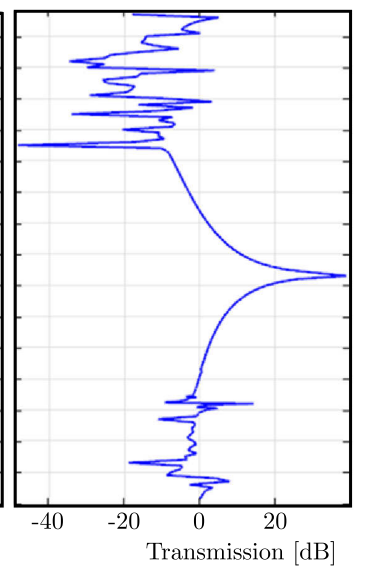

Fig. 2. Band structure of the proposed double panel structure and transmission power spectrums of the corresponding finite $8 \times 8$ system: (a) transmission power spectrum of flexural vibration when the response point and excitation point are picked on different plates; (b) band structure; (c) transmission power spectrum of longitudinal vibration when the response point and excitation point are picked on different plates; (d) transmission power spectrum of longitudinal vibration when both the response and excitation point are picked on the same plate

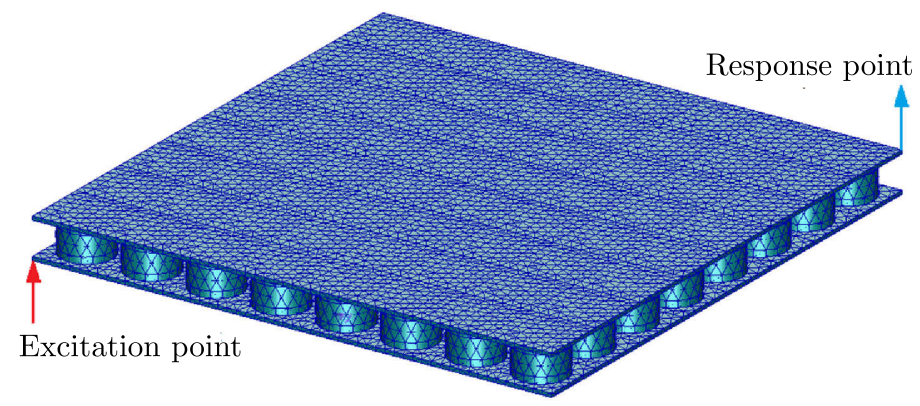

Fig. 3. Meshing of the finite locally resonant double panel structure made of $8 \times 8$ unit cells

hardly any frequency range of the attenuation can be observed. To reveal the mechanisms of typical phenomena displayed in transmission power spectrums of the locally resonant double panel structure, the displacement fields of the eigenmodes labeled in Fig. 2b are shown in Fig. 4.

For modes $B_{3}, B_{8}$ and $B_{9}$, the dominant vibration translating along the $z$ direction couples with the out-of-plane plate mode of the upper and lower plates. In mode $B_{3}$, the vibration energy is concentrated in the pillar with two plates static. Both in modes $B_{8}$ and $B_{9}$, the middle $\mathrm{Pb}$ layer of the pillar acts as a stationary layer. What is opposite, the two base plates achieve dynamic balance in the inverse flexural vibration in mode $B_{8}$ while the uniform flexural vibration of the two plates achieve dynamic balance in mode $B_{9}$, based on which, modes $B_{8}$ and $B_{9}$ are called as the symmetric flexural vibration mode and antisymmetric flexural vibration mode, respectively. As a result of the coupling, a partial flexural vibration band gap between $B_{3}$ and $B_{8}$ with a frequency interval $72 \mathrm{~Hz}-230 \mathrm{~Hz}$ is opened, which is why the big attenuation exists in the transmission power spectrum of the flexural vibration, as shown in Fig. 2a.

For modes $B_{1}$ and $B_{2}$, the dominant vibration rotating in the $x y$ plane couples with the out-of-plane shear deformation of the upper and lower plates. Both modes $B_{1}$ and $B_{2}$ concentrate the vibration energy in the pillar with the two stationary plates. As for modes $B_{4}-B_{7}$, they can be treated as the result of the coupling between the dominant vibration translating in the $x y$ plane and the in-plane shear deformation of the upper and lower plates. In all the four modes, the middle $\mathrm{Pb}$ layer of the pillar keeps stationary. Meanwhile, the uniform longitudinal 

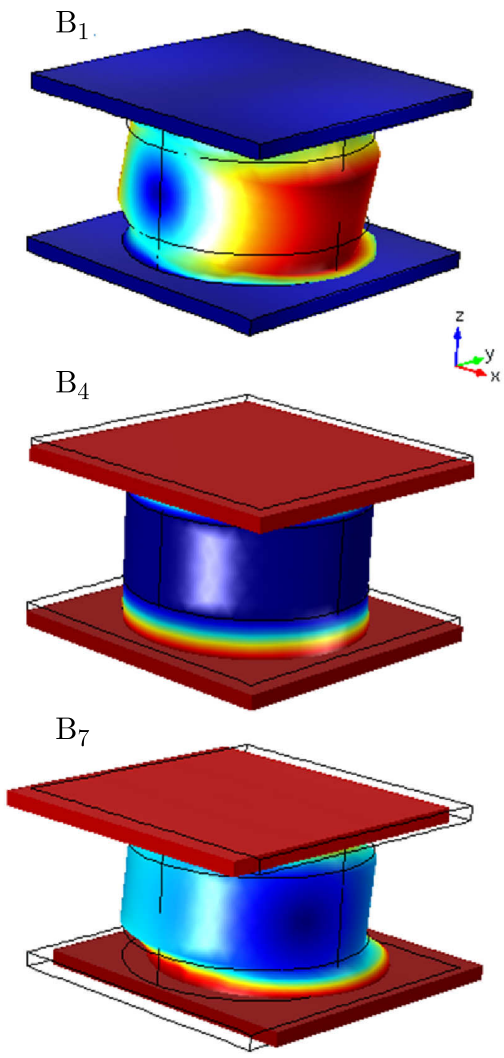
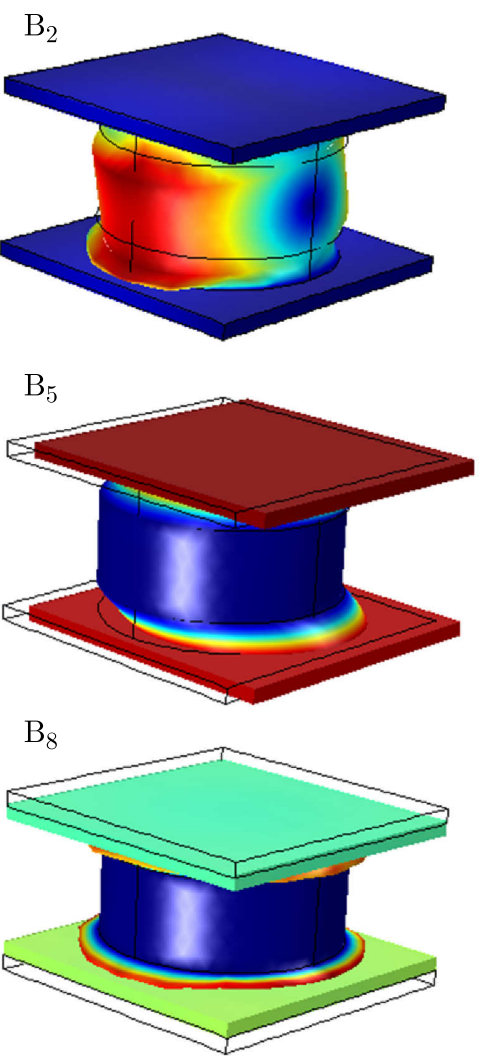
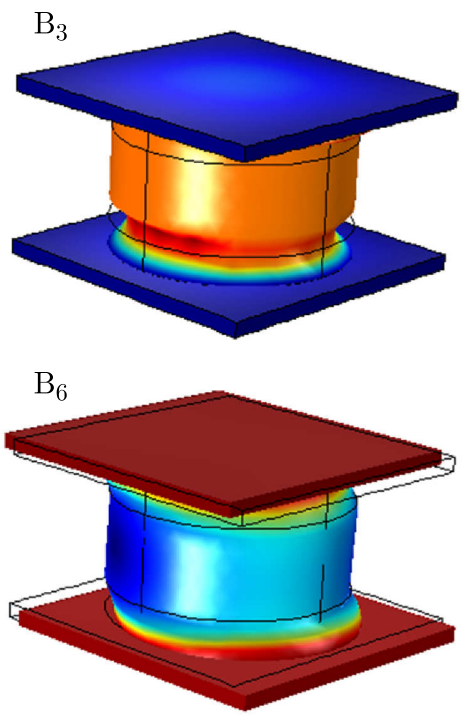

$\mathrm{B}_{9}$

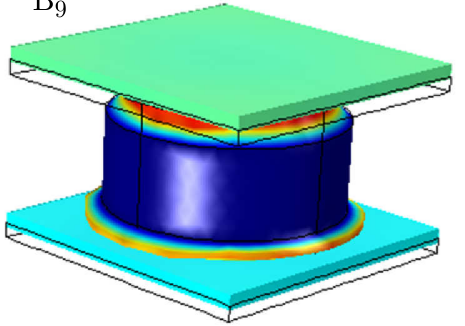

Fig. 4. The displacement fields of eigenmodes labeled in Fig. 2b

vibration of the lower and upper plates achieve dynamic balance in mode $B_{4}$ and $B_{5}$ while the two plates achieve dynamic balance in the inverse longitudinal vibration in mode $B_{6}$ and $B_{7}$. Similarly, modes $B_{4}-B_{5}$ and modes $B_{6}-B_{7}$ are called as the antisymmetric longitudinal vibration mode and symmetric longitudinal vibration mode, respectively. As a result of the couplings, a partial longitudinal vibration band gap with a frequency interval $66 \mathrm{~Hz}-83 \mathrm{~Hz}$ is opened. The corresponding attenuations existing in the transmission power spectrums of the longitudinal vibration shown in Figs. 2c and 2d are not obvious, which is consistent with the phenomenon described in (Xiao et al., 2008).

What should be noted is that the vibration of the upper plate is weakened because the vibration phases of the upper plate in the antisymmetric longitudinal vibration mode $B_{4}$ and the symmetric longitudinal vibration mode $B_{6}$ are inverse, as well as in $B_{5}$ and $B_{7}$. Based on this, the big attenuation displayed in the transmission spectrum of the longitudinal vibration shown in Fig. 2c can be understood. Meanwhile, the attenuation is absent in Fig. 2d because the lower plate vibrates strongly with the uniform phase. To further illustrate the attenuation characteristic, Fig. 5 shows a view of the vibration mode of the frequency located inside the frequency range of the attenuation. The calculation model is shown in Fig. 3. Here, the displacement excitation along three directions is imposed on the excitation point and the frequency is chosen as $f=140 \mathrm{~Hz}$. From Fig. 5, when both flexural and longitudinal excitations are imposed on the lower plate, none of the flexural and longitudinal vibrations can be propagated along the upper plate while only the longitudinal vibration can be propagated along the lower plate. In consequence, if the vibration source and the response area are on different sides of the double panel structure, the band gap with the starting frequency (higher frequency between $B_{2}$ and $B_{3}$ ) and cutoff frequency $B_{8}$ can be regarded as complete with the wide frequency range of attenuation in both flexural and longitudinal vibrations of the corresponding finite structure. 


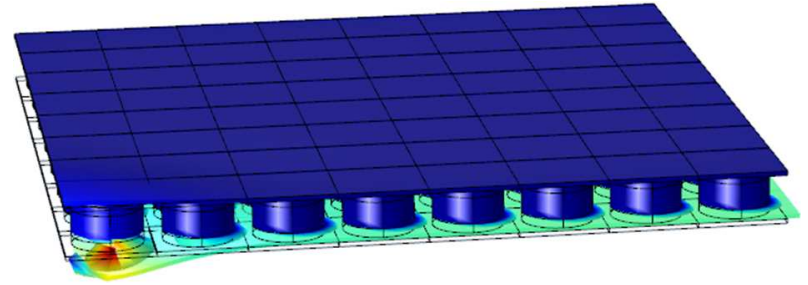

Fig. 5. The vibration mode of the frequency located inside the frequency range of attenuation

$$
(f=140 \mathrm{~Hz})
$$

\subsection{Comparison of double panel and single plate}

When the upper plate and the upper rubber layer of the pillar are taken away in Fig. 1b, the unit cell of the locally resonant single plate is formed. The band structure of the single plate is shown in Fig. 6a, where the parameters are same as those in the example shown in Fig. 2. To ease the comparison, the band structure of the locally resonant double panel, which is been shown in Fig. 2b, is replotted in Fig. 6b. In addition, the displacement fields of the eigenmodes labeled in Fig. 6a are shown in Fig. 7.

(a)

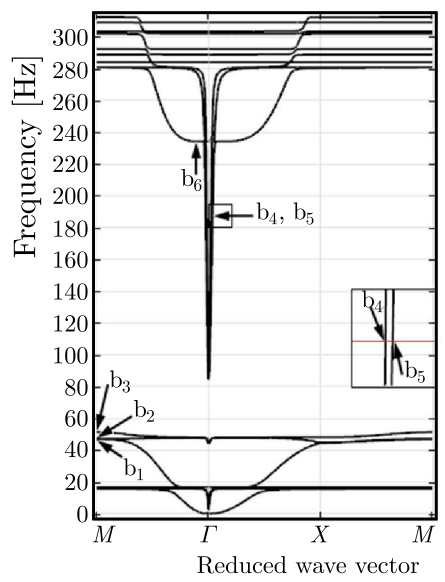

(b)

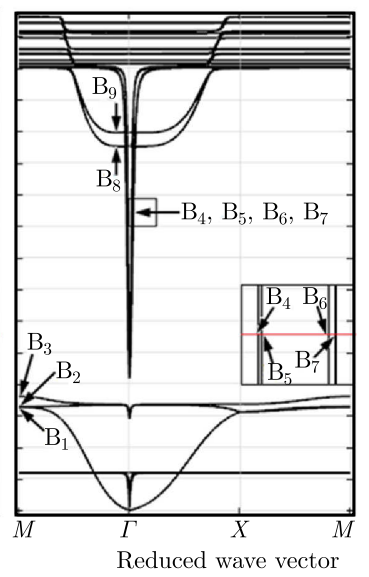

Fig. 6. Band structures of (a) the locally resonant single plate and (b) the locally resonant double panel with the same parameters
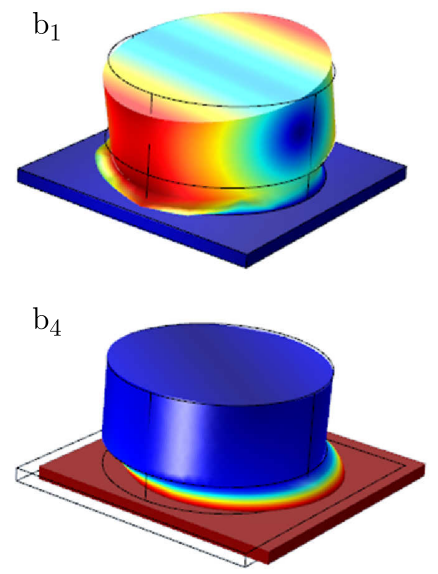
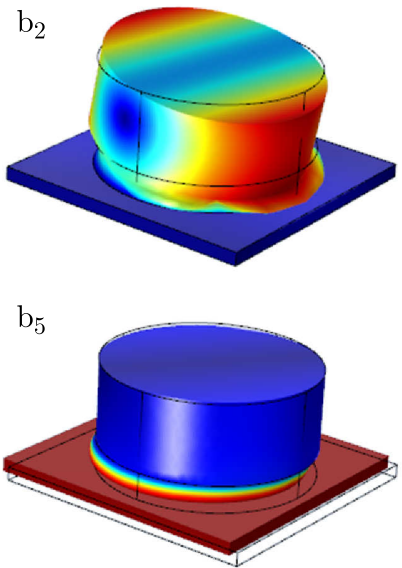

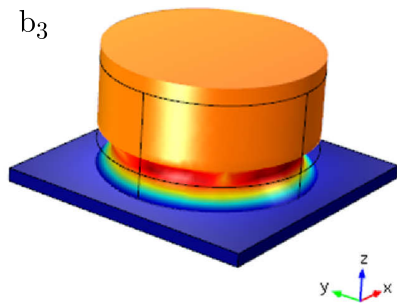

$\mathrm{b}_{6}$

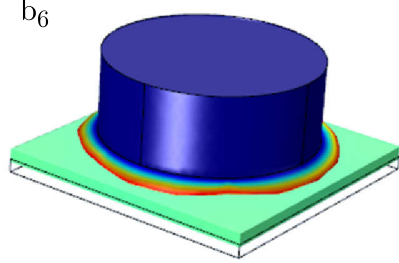

Fig. 7. The displacement fields of eigenmodes labeled in Fig. 6a 
Similarly, the coupling between the dominant vibration translating along the $z$ direction and the out-of-plane plate mode of the base plate as shown in modes $b_{3}$ and $b_{6}$ opens a partial flexural vibration band gap. Meanwhile, both the coupling between the dominant vibration rotating in the $x y$ plane and the out-of-plane shear deformation of base plate as shown in modes $b_{1}$ and $b_{2}$ and the coupling between the dominant vibration translating in the $x y$ plane and the in-plane shear deformation of the base plate as shown in modes $b_{5}$ open a partial longitudinal vibration band gap. As a result, a narrow complete band gap is opened in both flexural and longitudinal vibrations.

For modes $B_{1}-B_{3}$ and $b_{1}-b_{3}$, the base plates in all of them remain still and the dominant vibrations displayed in $B_{1}$ and $b_{1}$, dominant vibrations displayed in $B_{2}$ and $b_{2}$ as well as the dominant vibrations displayed in $B_{3}$ and $b_{3}$ are consistent, respectively. Here, the mass-spring models as shown in Fig. 8 are applied to help one to understand the vibration modes, which (a) and (b) with eigen frequencies $\sqrt{k / m}$ and $\sqrt{2 k / m}$ are regarded as simplified models of the vibrations in modes $b_{1}-b_{3}$ and $B_{1}-B_{3}$, respectively. According to such an analysis, the frequencies of modes $B_{1}-B_{3}$ should be $\sqrt{2}$ times larger than those of modes $b_{1}-b_{3}$. From Fig. 6 , the frequencies of modes $B_{1}-B_{3}$ are $65.3 \mathrm{~Hz}, 65.6 \mathrm{~Hz}$ and $72 \mathrm{~Hz}$, and the frequencies of modes $b_{1}-b_{3}$ are $46.9 \mathrm{~Hz}$, $47.2 \mathrm{~Hz}$ and $51 \mathrm{~Hz}$, which matches very well with $f_{B_{1}-B_{3}}=\sqrt{2} f_{b_{1}-b_{3}}$.
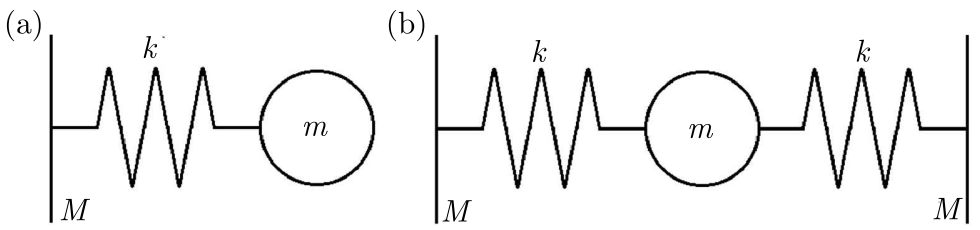

Fig. 8. The simplified mass-spring models applied to help understanding the vibration modes in (a) the single plate and (b) double panel

For modes $B_{4}-B_{9}$ and $b_{4}-b_{6}$, the middle $\mathrm{Pb}$ layer of the pillar in all of them stays stationary and the vibration modes displayed in $B_{4}, B_{6}$ and $b_{4}$, vibration modes displayed in $B_{5}, B_{7}$ and $b_{5}$ as well as vibration modes displayed in $B_{8}, B_{9}$ and $b_{6}$ are consistent, respectively. For each set of the corresponding vibration modes, the lower plate vibrates in the same direction as shown in the displacement fields. What should be noted is that the two vibration modes of the double panel are corresponding to each vibration mode of the single plate, which can be attributed to the effect of the upper plate. Besides, the vibration directions of the upper plate in the two different vibration modes of the double panel are opposite. Similarly, the mass-spring models shown in Fig. 8 can also be used to help understanding the vibration modes. Here, the mass $m$ is static and both the vibrations of the two base plates $M$ shown in Fig. $8 \mathrm{~b}$ can be treated as vibration of the single plate shown in Fig. 8a. Therefore, both the eigenfrequencies of the two models are the same $\sqrt{k / M}$. From Fig. 6 , the highest frequencies of the bands traveling modes $B_{4}-B_{9}$ are $280 \mathrm{~Hz}, 280 \mathrm{~Hz}, 281 \mathrm{~Hz}, 281 \mathrm{~Hz}, 282 \mathrm{~Hz}, 285 \mathrm{~Hz}$ and the highest frequencies of the bands traveling modes $b_{4}-b_{6}$ are $281 \mathrm{~Hz}, 281 \mathrm{~Hz}$ and $284 \mathrm{~Hz}$, which matches quite well with the analysis above. But in the low frequency range of the bands, traveling modes $B_{4}-B_{9}$, the middle $\mathrm{Pb}$ layer in the pillar cannot remain absolute still like in the high frequency range. The vibration amplitudes of the upper and lower plates have slight differences and the two vibration modes in the double panel corresponding to that of the single plate are not entirely same as shown in Fig. 4, so the bands traveling $B_{4}-B_{5}, B_{6}-B_{7}$ and $B_{8}-B_{9}$ are all divided unlike in the high frequency range, which can be understood as the division of the bands traveling $b_{4}, b_{5}$ and $b_{6}$ in the single plate, respectively.

In general, the band structures of the double panel and the single plate have much in common, and the overall trends between them displayed in Fig. 6 are similar. In addition, some pairs of the bands in the double panel structure can be treated as the division of the corresponding bands in the single plate. But the particular property displayed in the band structure of the 
double panel that a wide complete band gap is opened when the excitation and response areas are on different sides of the structure is incomparable.

\subsection{Influence of parameters on the band gap}

As is well known, the vibration source and the receiver are on the opposite sides of the double panel commonly. Thus, the band gap formed by $B_{2}, B_{3}$ and $B_{8}$ is considered and investigated below. To get the band gap with a low starting frequency and a wide band width, some geometrical parameters are picked to analyze the influences on the band gap. Here, the height of the rubber layer in the pillar $h_{1}$, height of the $\mathrm{Pb}$ layer in the pillar $h_{2}$, radius of the pillar $r$, thickness of the base plate $e$ and the lattice constant $a$ are chosen as the influencing parameters. During the study, the rest parameters are same as those in the example shown in Fig. 2a while considering the influence of one parameter on the starting frequency $f_{s}\left(B_{2}\right.$ or $\left.B_{3}\right)$, cutoff frequency $f_{c}\left(B_{8}\right)$ and band width $f_{w}$.

Figure 9 shows the influence of height of the rubber layer $h_{1}$ on $f_{2}, f_{3}, f_{8}$ and $f_{w}$. In the figure, $f_{w}$ equals to the difference between $f_{8}$ and $f_{3}$ as $f_{2}$ is always located under $f_{3}$. In addition, both $f_{3}$ and $f_{8}$ decrease with an increase in $h_{1}$, which can be understood by the model shown in Fig. 8b. In the model, the rubber layers are simplified as springs and $f_{3}$ and $f_{8}$ can be described by $\sqrt{2 k / m}$ and $\sqrt{k / M}$ qualitatively. Because the equivalent spring stiffness $k$ decreases with an increase in $h_{1}, f_{3}$ and $f_{8}$ decrease with an increase in height of the rubber layer. In addition, the equivalent mass of the $\mathrm{Pb}$ layer $m$ is larger than the equivalent mass of the base plate $M$ obviously, so the slope of $f_{3}$ is smaller than that of $f_{8}$ from $\sqrt{2 k / m}$ and $\sqrt{k / M}$ as shown in Fig. 9, which leads $f_{w}$ to decrease with an increase in $h_{1}$ as shown in Fig. 9.

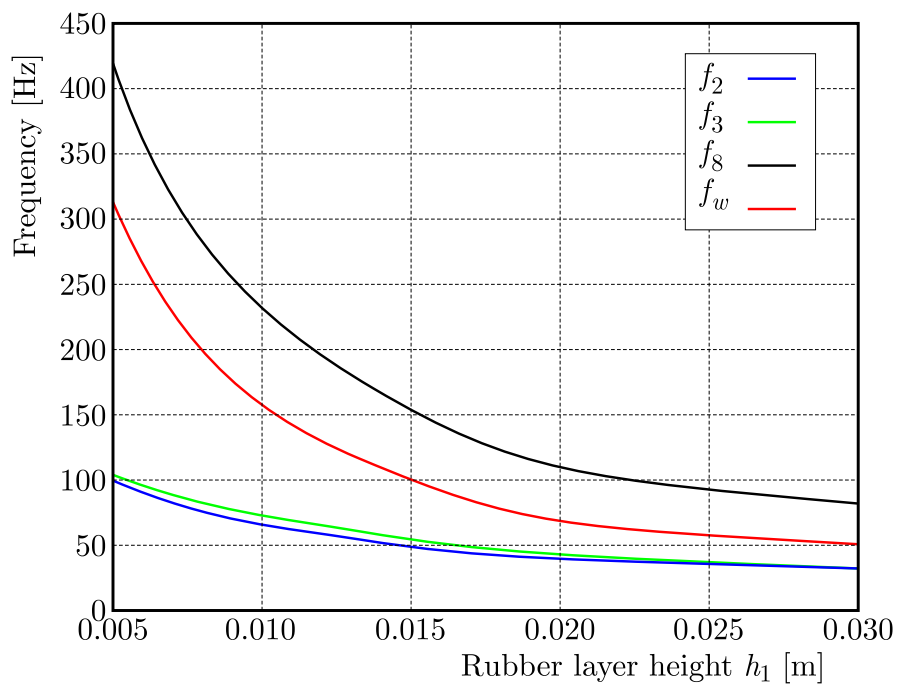

Fig. 9. The influence of the height of the rubber layer in the pillar $h_{1}$ on $f_{2}, f_{3}, f_{8}$ and $f_{w}$

Figure 10 shows the influence of the height of the $\mathrm{Pb}$ layer $h_{2}$ on $f_{2}, f_{3}, f_{8}$ and $f_{w}$. In the figure, $f_{w}$ also equals to the difference between $f_{8}$ and $f_{3}$. Moreover, both $f_{2}$ and $f_{3}$ decrease with an increase in $h_{2}$ because the equivalent mass of the $\mathrm{Pb}$ layer $m$ in the simplified model shown in Fig. $8 \mathrm{~b}$ is proportional to $h_{2} . f_{8}$ keeps nearly constant because the $\mathrm{Pb}$ layer is static in the vibration mode shown in Fig. 4. The variation trends of $f_{3}$ and $f_{8}$ lead to an increase in $f_{w}$ with an increase in $h_{2}$ as shown in the figure.

Figure 11 shows the influence of the radius of the pillar $r$ on $f_{2}, f_{3}, f_{8}$ and $f_{w}$. In the figure, $f_{w}$ is obtained by the difference between $f_{8}$ and $f_{3}$ as $f_{2}$ is always under $f_{3}$. Both $f_{2}$ and $f_{3}$ increase slowly with an increase in $r$, which can be attributed to an increase in the equivalent spring stiffness $k$ and the equivalent mass $m$ with an increase in $r$, and the larger slope of $k$ than 


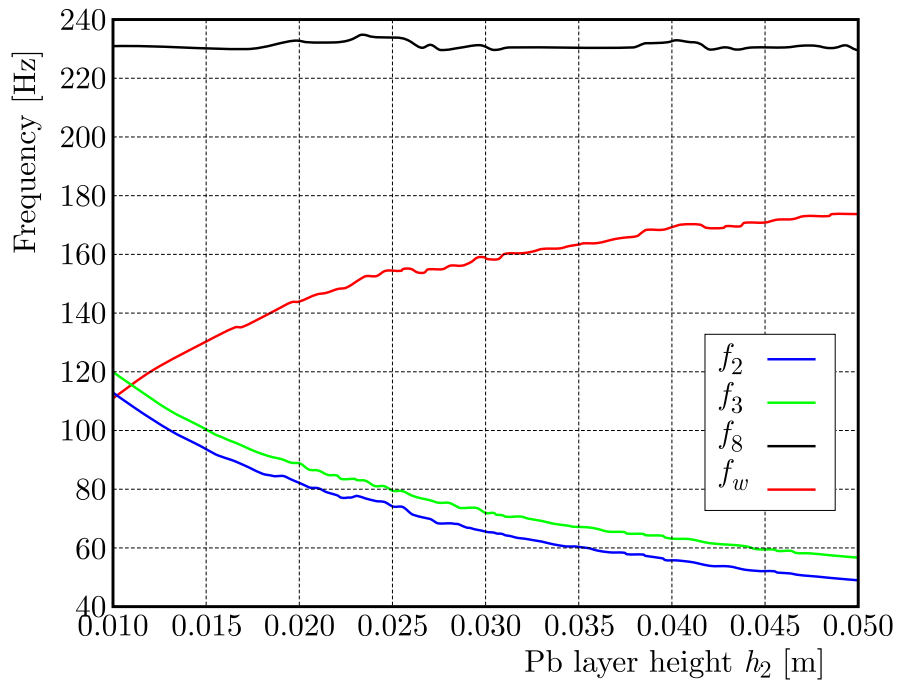

Fig. 10. The influences of the height of the $\mathrm{Pb}$ layer in the pillar $h_{2}$ on $f_{2}, f_{3}, f_{8}$ and $f_{w}$

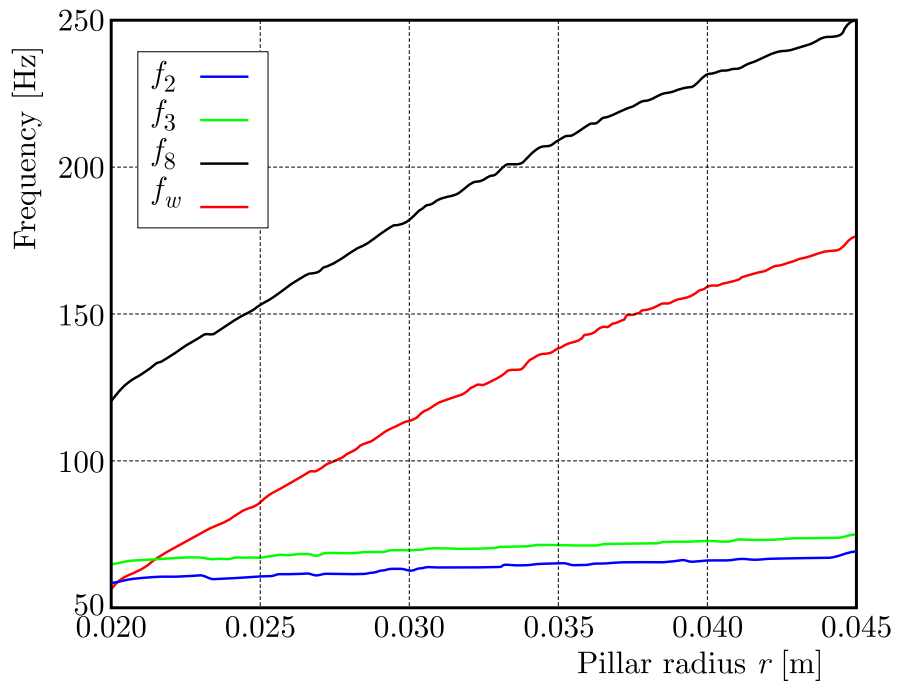

Fig. 11. The influences of the radius of the pillar $r$ on $f_{2}, f_{3}, f_{8}$ and $f_{w}$

that of $m$. Besides, $f_{8}$ increases rapidly with an increase in $r$ because the $\mathrm{Pb}$ layer is static here and only an increase in $k$ makes the slope of $f_{8}$ large. Hence, the rapid increase of $f_{8}$ and slow increase of $f_{3}$ lead $f_{w}$ to increase with an increase in $r$.

Figure 12 shows the influence of the thickness of the base plates $e$ on $f_{2}, f_{3}, f_{8}$ and $f_{w} . f_{w}$ is obtained by the difference between $f_{8}$ and the maximum of $f_{2}$ and $f_{3}$. From the figure, $f_{2}$ is larger when $e$ is less than $3.42 \cdot 10^{-3} \mathrm{~m}$ and $f_{3}$ is larger when $e$ is greater than $3.42 \cdot 10^{-3} \mathrm{~m}$. Both $f_{2}$ and $f_{3}$ increase with an increase in $e$, which can be understood well with the help of Fig. 13 . Figure 13 shows the displacement fields of modes $B_{2}$ and $B_{3}$ when $e$ takes the value of $0.003 \mathrm{~m}$ and $0.007 \mathrm{~m}$, respectively. From the figure, the base plates are vibrated but not absolutely static, and the vibration mode of the base plate is more easily displayed when $e$ equals to $0.003 \mathrm{~m}$ because of the smaller stiffness. Hence, the equivalent spring stiffness $k$ in the simplified model shown in Fig. 8b can be treated as a combined effect of both the base plates and the rubber layer in the pillar. Because the stiffness of the base plate increases with an increase in $e$, the equivalent spring stiffness $k$ increases, which results in an increase in $f_{2}$ and $f_{3}$. In addition, $f_{8}$ decreases with an increase in $e$ because the equivalent mass of the base plate $M$ increases with an increase in $e$. As a result, the decrease of $f_{8}$ and the increase of $f_{2}$ and $f_{3}$ lead $f_{w}$ to decrease with an increase in $e$. 


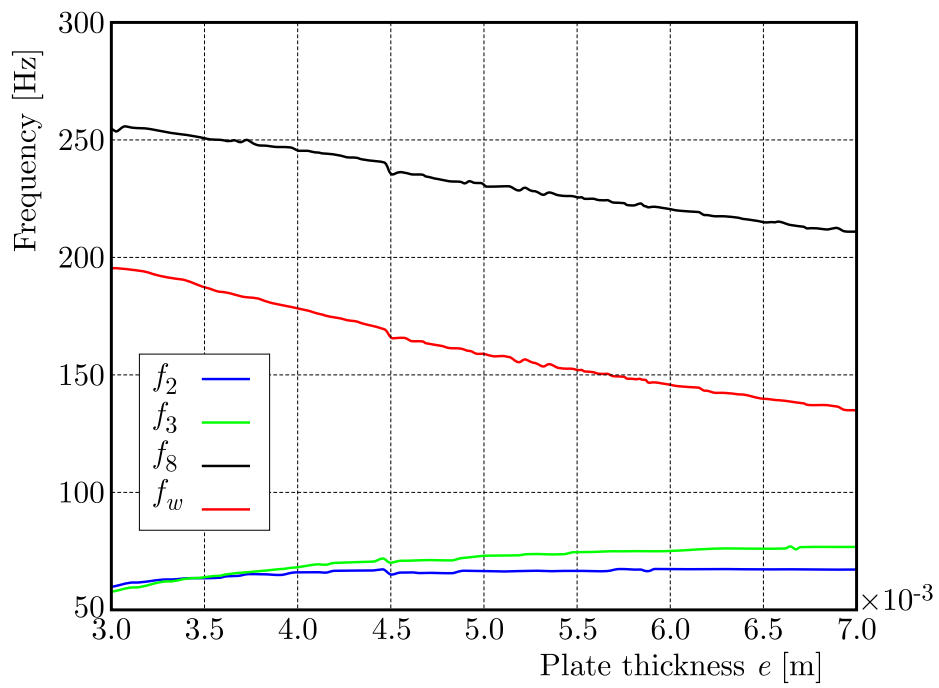

Fig. 12. The influences of the thickness of the base plates $e$ on $f_{2}, f_{3}, f_{8}$ and $f_{w}$
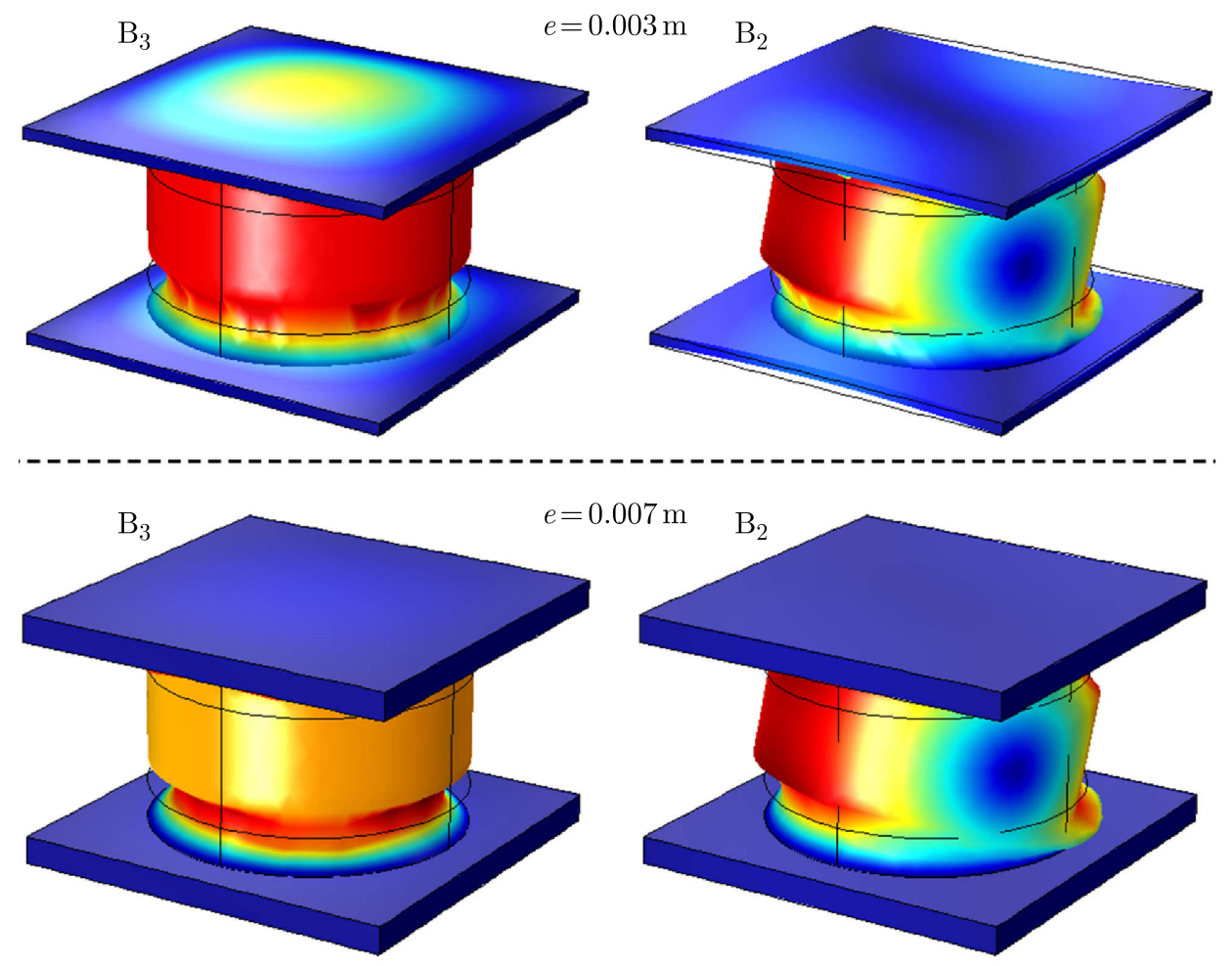

Fig. 13. The displacement fields of modes $B_{2}$ and $B_{3}$ when $e$ takes the value of $0.003 \mathrm{~m}$ and $0.007 \mathrm{~m}$, respectively

Figure 14 shows the influence of the lattice constant $a$ on $f_{2}, f_{3}, f_{8}$ and $f_{w} . f_{w}$ is obtained by the difference between $f_{8}$ and the maximum of $f_{2}$ and $f_{3}$. From the figure, $f_{3}$ is larger when $a$ is less than $0.132 \mathrm{~m}$ and $f_{2}$ is larger when $a$ is greater than $0.132 \mathrm{~m}$. $f_{2}$ keeps almost constant and $f_{3}$ decreases with an increase in $a$, which can also be understood with the help of the displacement fields of modes $B_{2}$ and $B_{3}$ when $a$ takes the value of $0.085 \mathrm{~m}$ and $0.2 \mathrm{~m}$, respectively, as shown in Fig. 15. From the figure, what can be concluded is that the stiffness of the base plate decreases with an increase in $a$. Besides, the variation of the stiffness of the base plate in mode $B_{2}$ is not big by comparing the displacement fields of modes $B_{2}$ when $a$ equals to $0.085 \mathrm{~m}$ and $0.2 \mathrm{~m}$ separately. Hence, the variation trends of $f_{2}$ and $f_{3}$ are explained. In addition, 
$f_{8}$ decreases with an increase in $a$ because the equivalent mass of the base plate $M$ increases with an increase in $e$. As a result, the rapid decrease of $f_{8}$ and the slow decrease of $f_{2}$ and $f_{3}$ shown in Fig. 14 lead $f_{w}$ to decrease with an increase in $a$.

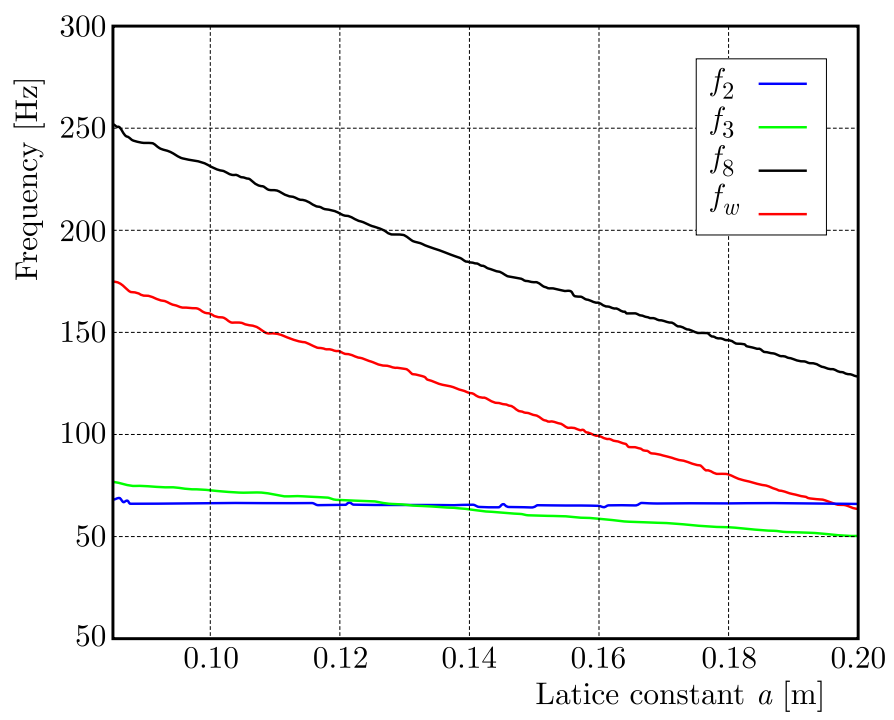

Fig. 14. The influences of the lattice constant $a$ on $f_{2}, f_{3}, f_{8}$ and $f_{w}$
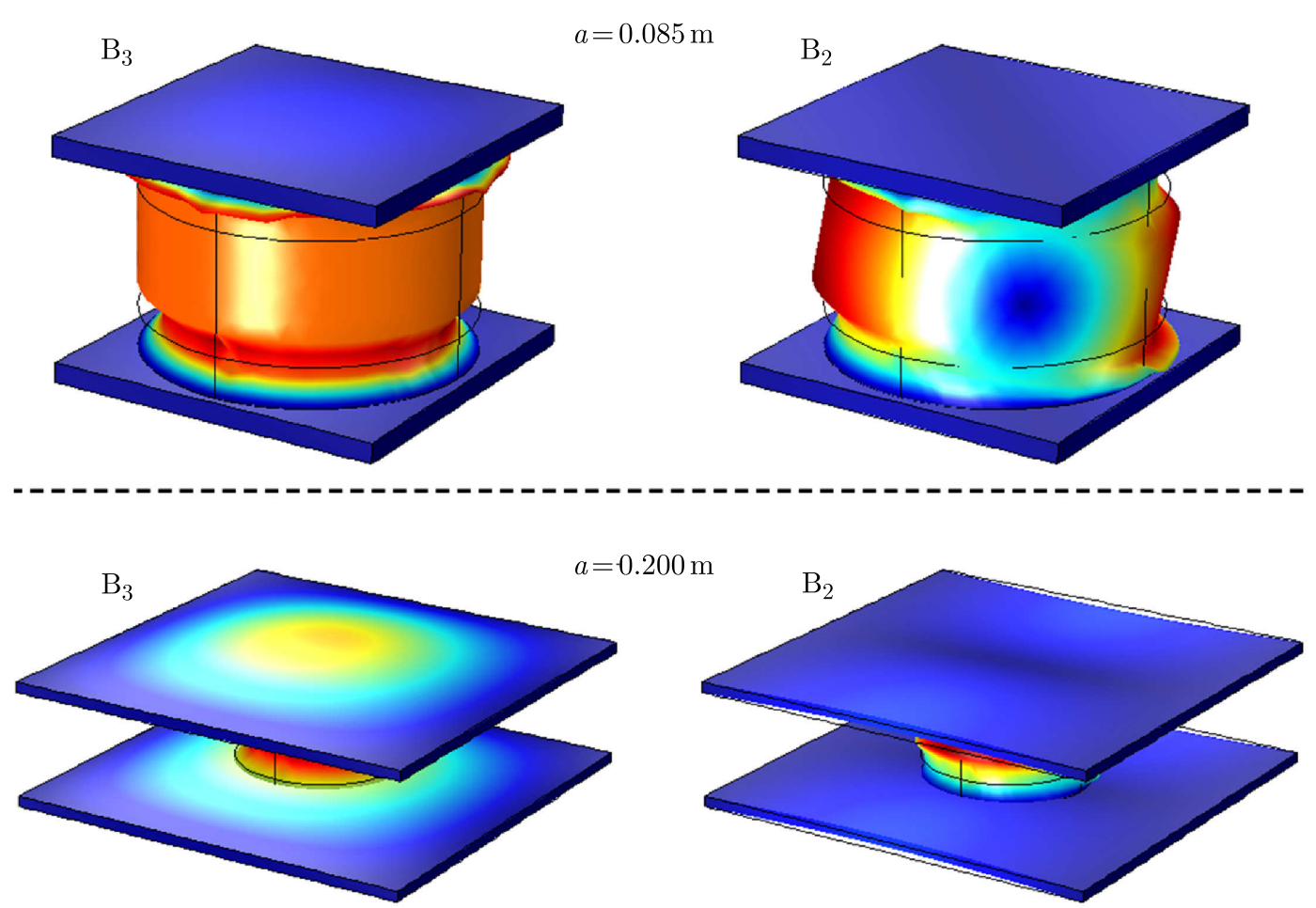

Fig. 15. The displacement fields of modes $B_{2}$ and $B_{3}$ when $a$ takes the value of $0.085 \mathrm{~m}$ and $0.2 \mathrm{~m}$, respectively

\section{Conclusions}

In this paper, we propose a three-component pillared phononic crystal double panel structure and study the bandgap properties of the structure by applying the finite element method. A complete band gap with a low starting frequency and a wide band width is opened according 
to the band structure and the transmission power spectrums when the vibration source and the receiver are on different sides of the structure, and the formation mechanisms of the band gap is revealed according to the displacement fields of the eigenmodes. By comparing the band structure of the double panel and that of the single plate, the bands of the double panel can be treated as the division of the corresponding bands of the single plate by the effect of the upper and lower plates. Besides, the influence of height of the rubber layer in the pillar, height of the $\mathrm{Pb}$ layer in the pillar, radius of the pillar, thickness of the base plate and the lattice constant on the band gap are studied and understood. In general, by increasing the height of the $\mathrm{Pb}$ layer or decreasing the thickness of the base plate, the starting frequency and band width of the band gap gets lower and wider. By decreasing the height of the rubber layer, the band width of the band gap gets wider, but increases the starting frequency. By increasing the radius of the pillar or decreasing the lattice constant, the band width of the band gap also gets wider, and the change in the starting frequency is not big. All the results of the investigation provide a new idea for restraining the vibration and reducing the noise of the low frequency region in the double panel structure.

\section{Acknowledgments}

This research has been supported by the National Natural Science Foundation of China through Grant No. 11172131, the Research Fund of State Key Laboratory of Mechanics and Control of Mechanical Structures (Nanjing University of Aeronautics and Astronautics) (Grant No. 0515G01) and the Foundation Research Project of Jiangsu Province (The Natural Science Fund No. 2015003-01).

\section{References}

1. Carneal J.P., Fuller C.R., 2004, An analytical and experimental investigation of active structural acoustic control of noise transmission through double panel systems, Journal of Sound and Vibration, 272, 3-5, 749-771

2. Goffaux C., Sánchez-Dehesa J., Lambin P., 2004, Comparison of the sound attenuation efficiency of locally resonant materials and elastic band-gap structures, Physical Review B, 70, 18, 184302

3. Hirsekorn M., Delsanto P.P., Batra N.K., Matic P., 2004, Modelling and simulation of acoustic wave propagation in locally resonant sonic materials, Ultrasonics, 42, 1, 231-235

4. Ho K.M., Cheng C.K., Yang Z., Zhang X.X., Sheng P., 2003, Broadband locally resonant sonic shields, Applied Physics Letters, 83, 26, 5566-5568

5. Hsu J.C., 2011, Local resonances-induced low-frequency band gaps in two-dimensional phononic crystal slabs with periodic stepped resonators, Journal of Physics D, Applied Physics, 44, 5, 55401-55409

6. Hsu J.C., Wu T.T., 2007, Lamb waves in binary locally resonant phononic plates with twodimensional lattices, Applied Physics Letters, 90, 20, 201904-201904-3

7. Li S., Chen T., Wang X., Li Y., Chen W., 2016, Expansion of lower-frequency locally resonant band gaps using a double-sided stubbed composite phononic crystals plate with composite stubs, Physics Letters A, 380, 25-26, 2167-2172

8. Li Y., Chen T., Wang X., Xi Y., Liang Q., 2015, Enlargement of locally resonant sonic band gap by using composite plate-type acoustic metamaterial, Physics Letters A, 379, 5, 412-416

9. Liu Z., Zhang X., Mao Y., Zhu Y., Yang Z., Chan C.T., Sheng P., 2000, Locally resonant sonic materials, Science, 289, 5485, 1734-1736

10. Ma J., Hou Z., Assouar B.M., 2014, Opening a large full phononic band gap in thin elastic plate with resonant units, Journal of Applied Physics, 115, 9, 093508-093508-5 
11. Oudich M., Li Y., Assouar B.M., Hou Z., 2010, A sonic band gap based on the locally resonant phononic plates with stubs, New Journal of Physics, 12, 2, 201-206

12. Oudich M., Senesi M., Assouar M.B., Ruzenne M., Sun J.-H., Vincent B., Hou Z., Wu T.-T., 2011, Experimental evidence of locally resonant sonic band gap in two-dimensional phononic stubbed plates, Physical Review B, 84, 16, 667-673

13. Pietrzko S.J., MaO Q., 2008, New results in active and passive control of sound transmission through double wall structures, Aerospace Science and Technology, 12, 1, 42-53

14. QIAN D., Shi Z., 2016, Bandgap properties in locally resonant phononic crystal double panel structures with periodically attached spring-mass resonators, Physics Letters A, 380, 41, 3319-3325

15. QIAN D, Shi Z., 2017, Using PWE/FE method to calculate the band structures of the semiinfinite beam-like PCs: Periodic in $z$-direction and finite in $x$ - $y$ plane, Physics Letters A, 381, 17 , $1516-1524$

16. Sainidou R., Stefanou N., Psarobas I.E., Modinos A., 2002, Scattering of elastic waves by a periodic monolayer of spheres, Physical Review B, 66, 2, 024303

17. Sigalas M.M., Economou E.N., 1992, Elastic and acoustic wave band structure, Journal of Sound and Vibration, 158, 2, 377-382

18. Vasseur J.O., Deymier P.A., Khelif A., Lambin Ph., Duafari-Rouhani B., Akjouj A., Dobrzynski L., Fettouhi N., Zemmouri J., 2002, Phononic crystal with low filling fraction and absolute acoustic band gap in the audible frequency range: A theoretical and experimental study, Physical Review E, 65, 5, 056608

19. Xiao W., Zeng G.W., Cheng Y.S., 2008, Flexural vibration band gaps in a thin plate containing a periodic array of hemmed discs, Applied Acoustics, 69, 3, 255-261

20. Xiao Y., Wen J., Wen X., 2012, Flexural wave band gaps in locally resonant thin plates with periodically attached spring-mass resonators, Journal of Physics D, Applied Physics, 45, 19, 195401-195412

21. Zhang X., Liu Z., Liu Y., Wu F., 2003, Elastic wave band gaps for three-dimensional phononic crystals with two structural units, Physics Letters A, 313, 5, 455-460

22. Zhao H.J., Guo H.W., Gao M.X., Liu R.-Q., Deng Z.-Q., 2016, Vibration band gaps in double-vibrator pillared phononic crystal plate, Journal of Applied Physics, 119, 1, 377 